\title{
ON SOME EQUIVALENT METRICS FOR BOUNDED OPERATORS ON HILBERT SPACE
}

\author{
FUAD KITTANEH
}

(Communicated by Palle E. T. Jorgensen)

\begin{abstract}
Several operator norm inequalities concerning the equivalence of some metrics for bounded linear operators on Hilbert space are given. In addition, some related inequalities for the Hilbert-Schmidt norm are presented.
\end{abstract}

\section{INTRODUCTION}

Let $H$ be a complex Hilbert space and let $C D(H)$ denote the family of closed, densely defined linear operators on $H$, and $B(H)$ the bounded members of $C D(H)$. For each $T \in C D(H)$, let $\Pi(T)$ denote the orthogonal projection of $H \oplus H$ onto the graph of $T$, and let $\alpha(T)$ denote the pure contraction defined by $\alpha(T)=T\left(1+T^{*} T\right)^{-1 / 2}$. The gap metric on $C D(H)$ is defined by $d(S, T)=\|\Pi(S)-\Pi(T)\|$ for all $S, T \in C D(H)$ (see [8, p. 197]). In [11], W. E. Kaufman introduced a metric $\delta$ on $C D(H)$ defined by $\delta(S, T)=$ $\|\alpha(S)-\alpha(T)\|$ for all $S, T \in C D(H)$ and he showed that this metric is stronger than the gap metric $d$ and not equivalent to it. He also stated that on $B(H)$ the gap metric $d$ is equivalent to the metric generated by the usual operator norm and he proved that the metric $\delta$ has this property.

The purpose of this paper is to present quantitative estimates to this effect. In $\S 2$, we present several operator norm inequalities to compare the metric $\delta$, the gap metric $d$, and the usual operator norm metric. In $\S 3$, we obtain $\delta$ estimates involving positive operators and operator monotone functions, and finally in $\S 4$, we remark how the inequalities of $\S \S 2,3$ can be extended to more general norms with an emphasis on the Hilbert-Schmidt norm.

To achieve our goal, we need the following lemmas. Lemmas 1 and 2 are concerned with the continuity of the square root function defined on positive operators. Lemma 3 is concerned with the continuity of the absolute value map, and Lemma 4 addresses the norm of operator matrices.

Received by the editors October 23, 1989.

1980 Mathematics Subject Classification (1985 Revision). Primary 47A30, 47A65, 47B10, 47B15.

Key words and phrases. Equivalent metrics, positive operator, operator monotone function, operator norm, Hilbert-Schmidt norm. 
Lemma 1 [14]. If $A, B \in B(H)$ are positive, then

$$
\|A-B\|^{2} \leq\left\|A^{2}-B^{2}\right\| .
$$

Lemma 2 [13]. If $A, B \in B(H)$ are positive and $A+B \geq c \geq 0$, then

$$
c\|A-B\| \leq\left\|A^{2}-B^{2}\right\| .
$$

Lemma 3 [16]. If $A, B \in B(H)$, then

$$
\||A|-|B|\| \leq\|A-B\|^{1 / 2}\|A+B\|^{1 / 2},
$$

where $|A|=\left(A^{*} A\right)^{1 / 2}$ is the absolute value of $A$.

Lemma 4 [3]. If $T=\left[\begin{array}{ll}A & B \\ C & D\end{array}\right] \in B(H \oplus H)$, then

$$
\|T\|^{2} \leq\|A\|^{2}+\|B\|^{2}+\|C\|^{2}+\|D\|^{2} .
$$

In addition to these lemmas we make repeated use of the operator identity:

$$
A B-C D=\frac{1}{2}(A-C)(B+D)+\frac{1}{2}(A+C)(B-D) .
$$

Consequently, for $S, T \in B(H)$, we have

$$
\left\|S^{*} S-T^{*} T\right\| \leq\|S-T\|\|S+T\|
$$

(see [16, Proof of Theorem 2.1]).

\section{Equivalent Metrics on $B(H)$}

In [11, Theorem 2], Kaufman proved that on $B(H)$ the metric $\delta$ and the usual operator norm metric are equivalent. There is a minor gap in the proof of Theorem 2 in [11] that can be filled by employing the continuity of the inversion map as demonstrated in the following two theorems.

Theorem 1. If $S, T \in B(H)$, then

$$
\delta(S, T) \leq\|S-T\|\left(1+\frac{1}{4}\|S+T\|^{2}\right) .
$$

Proof. For each $T \in B(H)$, let $\beta(T)=\left(1+T^{*} T\right)^{-1 / 2}$. Then $\beta(T)$ is a positive definite contraction; that is, $0<\beta(T) \leq 1, \alpha(T)=T \beta(T)$, and $\beta(T)=\left(1-\alpha(T)^{*} \alpha(T)\right)^{1 / 2}($ see $[9,11])$. 
For $S, T \in B(H)$, we have

$$
\begin{aligned}
\delta(S, T) & =\|\alpha(S)-\alpha(T)\| \\
& =\|S \beta(S)-T \beta(T)\| \\
& =\left\|\frac{1}{2}(S-T)(\beta(S)+\beta(T))+\frac{1}{2}(S+T)(\beta(S)-\beta(T))\right\| \\
& \leq\|S-T\|+\frac{1}{2}\|S+T\|\|\beta(S)-\beta(T)\| \\
& =\|S-T\|+\frac{1}{2}\|S+T\|\left\|\beta(S)\left(\beta(S)^{-1}-\beta(T)^{-1}\right) \beta(T)\right\| \\
& \leq\|S-T\|+\frac{1}{2}\|S+T\|\left\|\beta(S)^{-1}-\beta(T)^{-1}\right\| \\
& \leq\|S-T\|+\frac{1}{4}\|S+T\|\left\|\beta(S)^{-2}-\beta(T)^{-2}\right\| \quad(\text { Lemma 2) } \\
& =\|S-T\|+\frac{1}{4}\|S+T\|\left\|S^{*} S-T^{*} T\right\| \\
& \leq\|S-T\|+\frac{1}{4}\|S+T\|^{2}\|S-T\| \\
& =\|S-T\|\left(1+\frac{1}{4}\|S+T\|^{2}\right), \quad \text { as required. }
\end{aligned}
$$

Theorem 2. If $S, T \in B(H)$, then

$\|S-T\| \leq \frac{1}{4} \delta(S, T)\left(2\left\|\beta(S)^{-1}+\beta(T)^{-1}\right\|+\left\|\beta(S)^{-2}\right\|\left\|\beta(T)^{-2}\right\|\|\alpha(S)+\alpha(T)\|^{2}\right)$.

Proof. Since $T=\alpha(T) \beta(T)^{-1}$, it follows that

$$
\begin{aligned}
\|S-T\|= & \left\|\alpha(S) \beta(S)^{-1}-\alpha(T) \beta(T)^{-1}\right\| \\
= & \| \frac{1}{2}(\alpha(S)-\alpha(T))\left(\beta(S)^{-1}+\beta(T)^{-1}\right) \\
& +\frac{1}{2}(\alpha(S)+\alpha(T))\left(\beta(S)^{-1}-\beta(T)^{-1}\right) \| \\
\leq & \frac{1}{2} \delta(S, T)\left\|\beta(S)^{-1}+\beta(T)^{-1}\right\| \\
& +\frac{1}{2}\|\alpha(S)+\alpha(T)\|\left\|\beta(S)^{-1}-\beta(T)^{-1}\right\| \\
\leq & \frac{1}{2} \delta(S, T)\left\|\beta(S)^{-1}+\beta(T)^{-1}\right\| \\
& +\frac{1}{4}\|\alpha(S)+\alpha(T)\|\left\|\beta(S)^{-2}-\beta(T)^{-2}\right\| \quad(\text { Lemma 2) } \\
\leq & \frac{1}{2} \delta(S, T)\left\|\beta(S)^{-1}+\beta(T)^{-1}\right\| \\
& +\frac{1}{4}\left\|\beta(S)^{-2}\right\|\left\|\beta(T)^{-2}\right\|\|\alpha(S)+\alpha(T)\|\left\|\beta(S)^{2}-\beta(T)^{2}\right\| .
\end{aligned}
$$

Since $\left\|\beta(S)^{2}-\beta(T)^{2}\right\|=\left\|\alpha(S)^{*} \alpha(S)-\alpha(T)^{*} \alpha(T)\right\| \leq \delta(S, T)\|\alpha(S)+\alpha(T)\|$, it follows that

$$
\begin{aligned}
\|S-T\| \leq & \frac{1}{2} \delta(S, T)\left\|\beta(S)^{-1}+\beta(T)^{-1}\right\| \\
& +\frac{1}{4}\left\|\beta(S)^{-2}\right\|\left\|\beta(T)^{-2}\right\|\|\alpha(S)+\alpha(T)\|^{2} \delta(S, T) .
\end{aligned}
$$

This completes the proof of the theorem.

It has been stated (without proof) in [11] that on $B(H)$ the gap metric $d$ is equivalent to the usual operator norm metric. This is an immediate consequence of the results of this section. 
Theorem 3. If $S, T \in B(H)$, then

$$
\begin{aligned}
d^{2}(S, T) \leq & 2 \delta^{2}(S, T)\|\alpha(S)+\alpha(T)\|^{2} \\
& +2\left(\delta(S, T)+\frac{1}{2}\|\alpha(S)+\alpha(T)\|^{3 / 2} \delta^{1 / 2}(S, T)\right)^{2} .
\end{aligned}
$$

Proof. It is known [9, Remark, p. 532] that the projection-function $\Pi$ has an operator matrix representation given by

$$
\Pi(T)=\left[\begin{array}{cc}
\beta(T)^{2} & \beta(T) \alpha(T)^{*} \\
\alpha(T) \beta(T) & \alpha(T) \alpha(T)^{*}
\end{array}\right] .
$$

Using this representation together with Lemma 4 , we get

$$
\begin{aligned}
d^{2}(S, T)= & \|\Pi(S)-\Pi(T)\|^{2} \\
\leq & \left\|\beta(S)^{2}-\beta(T)^{2}\right\|^{2}+2\|\alpha(S) \beta(S)-\alpha(T) \beta(T)\|^{2} \\
& +\left\|\alpha(S) \alpha(S)^{*}-\alpha(T) \alpha(T)^{*}\right\|^{2} \\
= & \left\|\alpha(S)^{*} \alpha(S)-\alpha(T)^{*} \alpha(T)\right\|^{2}+2\|\alpha(S) \beta(S)-\alpha(T) \beta(T)\|^{2} \\
& +\left\|\alpha(S) \alpha(S)^{*}-\alpha(T) \alpha(T)^{*}\right\|^{2} .
\end{aligned}
$$

Now observe that both

$$
\left\|\alpha(S)^{*} \alpha(S)-\alpha(T)^{*} \alpha(T)\right\|^{2}
$$

and

$$
\left\|\alpha(S) \alpha(S)^{*}-\alpha(T) \alpha(T)^{*}\right\|^{2}
$$

are majorized by $\delta^{2}(S, T)\|\alpha(S)+\alpha(T)\|^{2}$. Observe also that

$$
\begin{aligned}
\| \alpha(S) & \beta(S)-\alpha(T) \beta(T) \| \\
\quad & \leq \frac{1}{2} \delta(S, T)\|\beta(S)+\beta(T)\|+\frac{1}{2}\|\alpha(S)+\alpha(T)\|\|\beta(S)-\beta(T)\| \\
& \leq \delta(S, T)+\frac{1}{2}\|\alpha(S)+\alpha(T)\|\|\beta(S)-\beta(T)\| \\
& \leq \delta(S, T)+\frac{1}{2}\|\alpha(S)+\alpha(T)\|\left\|\beta(S)^{2}-\beta(T)^{2}\right\|^{1 / 2}(\text { Lemma 1) } \\
& \leq \delta(S, T)+\frac{1}{2}\|\alpha(S)+\alpha(T)\|^{3 / 2} \delta^{1 / 2}(S, T) .
\end{aligned}
$$

In view of these observations we have

$$
\begin{aligned}
d^{2}(S, T) \leq & 2 \delta^{2}(S, T)\|\alpha(S)+\alpha(T)\|^{2}+2(\delta(S, T) \\
& \left.+\frac{1}{2}\|\alpha(S)+\alpha(T)\|^{3 / 2} \delta^{1 / 2}(S, T)\right)^{2}
\end{aligned}
$$

as required.

It can easily be seen that for $S, T \in B(H)$ we have $\alpha(S)^{*} \alpha(S)-\alpha(T)^{*} \alpha(T)=$ $\beta(S)^{2}\left(S^{*} S-T^{*} T\right) \beta(T)^{2}$. It follows from this identity that

$$
\left\|\alpha(S)^{*} \alpha(S)-\alpha(T)^{*} \alpha(T)\right\| \leq\left\|S^{*} S-T^{*} T\right\| .
$$

This estimate enables us to show that for $S, T \in B(H)$ we have

$$
d^{2}(S, T) \leq\|S-T\|^{2}\left(2+4\|S+T\|^{2}+\frac{1}{2}\|S+T\|^{4}\right) .
$$


For $T \in B(H)$ we have $\|\beta(T)\| \leq 1$ and $\|\alpha(T)\|<1$ (see [9]). However, $\|\alpha(T) \beta(T)\| \leq \frac{1}{2}$, which is proved as follows.

$$
\begin{aligned}
|\alpha(T) \beta(T)|^{2} & =\beta(T) \alpha(T)^{*} \alpha(T) \beta(T) \\
& =T^{*} T\left(1+T^{*} T\right)^{-2} \leq \frac{1}{4} .
\end{aligned}
$$

Hence $\|\alpha(T) \beta(T)\| \leq \frac{1}{2}$. This estimate is needed for the proof of the following theorem.

Theorem 4. If $S, T \in B(H)$, then

$$
\delta(S, T) \leq \frac{1}{4} d(S, T)\left(2\left\|\beta(S)^{-1}+\beta(T)^{-1}\right\|+\left\|\beta(S)^{-2}\right\|\left\|\beta(T)^{-2}\right\|\right) .
$$

Proof. Since $\alpha(T)=\alpha(T) \beta(T) \beta(T)^{-1}$, it follows that

$$
\begin{aligned}
\delta(S, T)= & \left\|\alpha(S) \beta(S) \beta(S)^{-1}-\alpha(T) \beta(T) \beta(T)^{-1}\right\| \\
\leq & \frac{1}{2}\|\alpha(S) \beta(S)-\alpha(T) \beta(T)\|\left\|\beta(S)^{-1}+\beta(T)^{-1}\right\| \\
& +\frac{1}{2}\|\alpha(S) \beta(S)+\alpha(T) \beta(T)\|\left\|\beta(S)^{-1}-\beta(T)^{-1}\right\| \\
\leq & \frac{1}{2} d(S, T)\left\|\beta(S)^{-1}+\beta(T)^{-1}\right\|+\frac{1}{2}\left\|\beta(S)^{-1}-\beta(T)^{-1}\right\| \\
\leq & \frac{1}{2} d(S, T)\left\|\beta(S)^{-1}+\beta(T)^{-1}\right\|+\frac{1}{4}\left\|\beta(S)^{-2}-\beta(T)^{-2}\right\| \quad(\text { Lemma 2) } \\
\leq & \frac{1}{2} d(S, T)\left\|\beta(S)^{-1}+\beta(T)^{-1}\right\| \\
& +\frac{1}{4}\left\|\beta(S)^{-2}\right\|\left\|\beta(T)^{-2}\right\|\left\|\beta(S)^{2}-\beta(T)^{2}\right\| \\
\leq & \frac{1}{2} d(S, T)\left\|\beta(S)^{-1}+\beta(T)^{-1}\right\|+\frac{1}{4}\left\|\beta(S)^{-2}\right\|\left\|\beta(T)^{-2}\right\| d(S, T),
\end{aligned}
$$

which completes the proof of the theorem.

Using the observation that $T=\alpha(T) \beta(T) \beta(T)^{-2}$, it can easily be shown that for $S, T \in B(H)$, we have

$$
\|S-T\| \leq \frac{1}{2} d(S, T)\left(\left\|\beta(S)^{-2}+\beta(T)^{-2}\right\|+\left\|\beta(S)^{-2}\right\|\left\|\beta(T)^{-2}\right\|\right) .
$$

In [11, Theorem 5], Kaufman proved that if $\left\{S_{n}\right\}$ is a sequence in $C D(H)$ and $T \in C D(H)$ is such that $\delta\left(S_{n}, T\right) \rightarrow 0$ as $n \rightarrow \infty$, then $\delta\left(\left|S_{n}\right|,|T|\right) \rightarrow 0$ as $n \rightarrow \infty$. For bounded operators this is an immediate consequence of the following estimate.

Theorem 5. If $S, T \in B(H)$, then

$$
\delta(|S|,|T|) \leq\|\alpha(S)-\alpha(T)\|^{1 / 2}\|\alpha(S)+\alpha(T)\|^{1 / 2} .
$$

Proof. Since $\alpha(|T|)=|\alpha(T)|$ (see [9, Lemma 3]), it follows that

$$
\begin{aligned}
\delta(|S|,|T|) & =\|\alpha(|S|)-\alpha(|T|)\| \\
& =\||\alpha(S)|-|\alpha(T)|\| \\
& \leq\|\alpha(S)-\alpha(T)\|^{1 / 2}\|\alpha(S)+\alpha(T)\|^{1 / 2} \quad(\text { Lemma 3). }
\end{aligned}
$$




\section{3. $\delta$-ESTIMATES FOR POSITIVE OPERATORS}

\section{AND OPERATOR MONOTONE FUNCTIONS}

Recall that a real-valued continuous function $f$ on $[0, \infty)$ is said to be operator monotone if for any positive operators $A, B \in B(H)$, the relation $A \leq B$ always implies $f(A) \leq f(B)$. It is well known that $f(t)=t^{r}$ is operator monotone for $0<r \leq 1$ (see [1]).

It has been shown in [16, Theorem 2.3] that if $A, B \in B(H)$ are positive, then for any operator monotone function $f$ with $f(0)=0$ we have

$$
\|f(A)-f(B)\| \leq f(\|A-B\|) .
$$

In the same spirit we have the following related result for the metric $\delta$.

Theorem 6. If $A, B \in B(H)$ are positive, then for any real-valued continuous function $f$ on $[0, \infty)$ with $f(0)=0$ and $f^{2}$ operator monotone, we have $\delta(f(A), f(B)) \leq f(\|A-B\|)$.

Proof. We have

$$
\begin{aligned}
\delta(f(A), f(B)) & =\left\|f(A)\left(1+f(A)^{2}\right)^{-1 / 2}-f(B)\left(1+f(B)^{2}\right)^{-1 / 2}\right\| \\
& \leq\left\|f(A)^{2}\left(1+f(A)^{2}\right)^{-1}-f(B)^{2}\left(1+f(B)^{2}\right)^{-1}\right\|^{1 / 2} \quad(\text { Lemma 1) } \\
& =\left\|\left(1+f(A)^{2}\right)^{-1}\left(f(A)^{2}-f(B)^{2}\right)\left(1+f(B)^{2}\right)^{-1}\right\|^{1 / 2} \\
& \leq\left\|f(A)^{2}-f(B)^{2}\right\|^{1 / 2} \\
& \leq f(\|A-B\|) \quad\left(\left[16, \text { Theorem 2.3] applied to } f^{2}\right) .\right.
\end{aligned}
$$

The following corollary is an important special case of Theorem 6 .

Corollary 1. If $A, B \in B(H)$ are positive, then for any real number $r, 0<r \leq$ $\frac{1}{2}$, we have

$$
\delta\left(A^{r}, B^{r}\right) \leq\|A-B\|^{r} .
$$

It should be noted that if $f$ is a nonnegative continuous function on $[0, \infty)$ such that $f^{2}$ is operator monotone, then $f$ is also operator monotone. Of course, the converse is not true. If we merely assume in Theorem 6 that $f$ is operator monotone, then we have the following weaker result.

Theorem 7. If $A, B \in B(H)$ are positive, then for any operator monotone function $f$ on $[0, \infty)$ with $f(0)=0$ we have

$$
\delta(f(A), f(B)) \leq\left(2 f(\|A-B\|) f\left(\frac{1}{2}\|A+B\|\right)\right)^{1 / 2} .
$$

Proof. We have

$$
\begin{aligned}
\delta(f(A), f(B)) & \leq\left\|f(A)^{2}-f(B)^{2}\right\|^{1 / 2} \quad(\text { from the proof of Theorem 6) } \\
& \leq\|f(A)-f(B)\|^{1 / 2}\|f(A)+f(B)\|^{1 / 2} \\
& \leq f(\|A-B\|)^{1 / 2}\|f(A)+f(B)\|^{1 / 2} \quad[16, \text { Theorem 2.3]. }
\end{aligned}
$$


But it is known that every operator monotone function is operator concave (see [1]), and hence $\frac{1}{2}\left(f(A)+f(B) \leq f\left(\frac{1}{2}(A+B)\right)\right.$. Therefore we have

$$
\begin{aligned}
\delta(f(A), f(B)) & \leq\left(2 f(\|A-B\|) \| f\left(\frac{1}{2}(A+B) \|\right)^{1 / 2}\right. \\
& \leq\left(2 f(\|A-B\|) f\left(\frac{1}{2}\|A+B\|\right)\right)^{1 / 2} .
\end{aligned}
$$

Corollary 2. If $A, B \in B(H)$ are positive, then for any real number $r, 0<r \leq$ 1 , we have

$$
\delta\left(A^{r}, B^{r}\right) \leq 2^{1-r / 2}\|A-B\|^{r / 2}\|A+B\|^{r / 2} .
$$

In particular $\delta(A, B) \leq\|A-B\|^{1 / 2}\|A+B\|^{1 / 2}$.

For arbitrary operators $S, T \in B(H)$ we have the following generalization of Corollary 2 for the case $r=1$, which is related to Theorem 5 .

Corollary 3. If $S, T \in B(H)$, then

$$
\delta(|S|,|T|) \leq\|S-T\|^{1 / 2}\|S+T\|^{1 / 2} .
$$

Proof. By Corollary 1, applied to the positive operators $S^{*} S$ and $T^{*} T, r=\frac{1}{2}$, we have

$$
\begin{aligned}
\delta(|S|,|T|) & \leq\left\|S^{*} S-T^{*} T\right\|^{1 / 2} \\
& \leq\|S-T\|^{1 / 2}\|S+T\|^{1 / 2} .
\end{aligned}
$$

It should be noted that an alternative proof of Corollary 3 can be provided. This proof is based on the fact that $\alpha(|T|)=|\alpha(T)|$, Lemma 1, and the relation $\alpha(S)^{*} \alpha(S)-\alpha(T)^{*} \alpha(T)=\beta(S)^{2}\left(S^{*} S-T^{*} T\right) \beta(T)^{2}$.

\section{INEQUALITIES FOR THE HILBERT-SCHMIDT NORM}

We remark that inequalities similar to those given in $\S \S 2,3$ can be proved for certain unitarily invariant norms including the Schatten $p$-norms (see [17]). This is due to the availability of inequalities in these norms similar to those given in Lemmas 1-4 and [16, Theorem 2.3]. For such inequalities the reader is referred to $[2,4-7,15]$ and references therein.

Besides the usual operator norm, the Hilbert-Schmidt norm is of particular importance among the unitarily invariant norms. The following improvements of Theorems 1 and 3 are available for the Hilbert-Schmidt norm.

Theorem 8. If $S, T \in B(H)$, then

$$
\|\alpha(S)-\alpha(T)\|_{2} \leq\|S-T\|_{2},
$$

where $\|\cdot\|_{2}$ denotes the Hilbert-Schmidt norm. In particular if $S-T$ is in the Hilbert-Schmidt class, then so is $\alpha(S)-\alpha(T)$.

Proof. We start by observing that $\left|s / \sqrt{1+s^{2}}-t / \sqrt{1+t^{2}}\right| \leq|s-t|$ for all real numbers $s$ and $t$. Therefore if we first assume that $S$ and $T$ are selfadjoint operators, then by [12] we get $\|\alpha(S)-\alpha(T)\|_{2} \leq\|S-T\|_{2}$. 
The general case follows by applying the selfadjoint case to the selfadjoint operators

$$
\widetilde{S}=\left[\begin{array}{cc}
0 & S^{*} \\
S & 0
\end{array}\right] \text { and } \tilde{T}=\left[\begin{array}{cc}
0 & T^{*} \\
T & 0
\end{array}\right]
$$

defined on $H \oplus H$. Using the fact (see [10]) that $\alpha\left(T^{*}\right)=\alpha(T)^{*}$ together with some matrix computations, we obtain

$$
\alpha(\widetilde{S})=\left[\begin{array}{cc}
0 & \alpha(S)^{*} \\
\alpha(S) & 0
\end{array}\right] \quad \text { and } \quad \alpha(\widetilde{T})=\left[\begin{array}{cc}
0 & \alpha(T)^{*} \\
\alpha(T) & 0
\end{array}\right]
$$

Now

$$
\|\widetilde{S}-\widetilde{T}\|_{2}^{2}=2\|S-T\|_{2}^{2}
$$

and

$$
\|\alpha(\widetilde{S})-\alpha(\widetilde{T})\|_{2}^{2}=2\|\alpha(S)-\alpha(T)\|_{2}^{2} .
$$

Since $\|\alpha(\widetilde{S})-\alpha(\widetilde{T})\|_{2}^{2} \leq\|\widetilde{S}-\widetilde{T}\|_{2}^{2}$, it follows that $\|\alpha(S)-\alpha(T)\|_{2} \leq\|S-T\|_{2}$ as required.

Theorem 9. If $S, T \in B(H)$, then

$$
\|\Pi(S)-\Pi(T)\|_{2}^{2} \leq 2\|S-T\|_{2}^{2}\left(\|\alpha(S)+\alpha(T)\|^{2}+1\right) .
$$

In particular if $S-T$ is in the Hilbert-Schmidt class, then so is $\Pi(S)-\Pi(T)$. Proof. Using the matrix representation of $\Pi$, as in the proof of Theorem 3, we have

$$
\begin{aligned}
\|\Pi(S)-\Pi(T)\|_{2}^{2}= & \left\|\beta(S)^{2}-\beta(T)\right\|_{2}^{2}+2\|\alpha(S) \beta(S)-\alpha(T) \beta(T)\|_{2}^{2} \\
& +\left\|\alpha(S) \alpha(S)^{*}-\alpha(T) \alpha(T)^{*}\right\|_{2}^{2} \\
= & \left\|\alpha(S)^{*} \alpha(S)-\alpha(T)^{*} \alpha(T)\right\|_{2}^{2}+2\left\|S \beta(S)^{2}-T \beta(T)^{2}\right\|_{2}^{2} \\
& +\left\|\alpha(S) \alpha(S)^{*}-\alpha(T) \alpha(T)^{*}\right\|_{2}^{2} .
\end{aligned}
$$

Now, note that both

$$
\left\|\alpha(S)^{*} \alpha(S)-\alpha(T)^{*} \alpha(T)\right\|_{2}^{2}
$$

and

$$
\left\|\alpha(S) \alpha(S)^{*}-\alpha(T) \alpha(T)^{*}\right\|_{2}^{2}
$$

are majorized by $\|\alpha(S)-\alpha(T)\|_{2}^{2}\|\alpha(S)+\alpha(T)\|^{2}$, and hence, by Theorem 8 , this is in turn majorized by $\|S-T\|_{2}^{2}\|\alpha(S)+\alpha(T)\|^{2}$.

Finally, by noting that $\left|s /\left(1+s^{2}\right)-t /\left(1+t^{2}\right)\right| \leq|s-t|$ for all real numbers $s$ and $t$, an argument similar to the one used in the proof of Theorem 8 can be presented here to show that $\left\|S \beta(S)^{2}-T \beta(T)^{2}\right\|_{2} \leq\|S-T\|_{2}$. Therefore, we conclude that

$$
\|\Pi(S)-\Pi(T)\|_{2}^{2} \leq 2\|S-T\|_{2}^{2}\|\alpha(S)+\alpha(T)\|^{2}+2\|S-T\|_{2}^{2},
$$

which completes the proof of the theorem. 
Once again if we use the relation

$$
\alpha(S)^{*} \alpha(S)-\alpha(T)^{*} \alpha(T)=\beta(S)^{2}\left(S^{*} S-T^{*} T\right) \beta(T)^{2},
$$

then we can also show that for $S, T \in B(H)$, we have

$$
\|\Pi(S)-\Pi(T)\|_{2}^{2} \leq 2\|S-T\|_{2}^{2}\left(\|S+T\|^{2}+1\right) .
$$

Whenever this estimate is compared with the estimate given in Theorem 9, one should keep in mind that $\|\alpha(S)+\alpha(T)\|<2$.

We have seen from the proof of Theorem 9 that if $S, T \in B(H)$, then

$$
\left\|S \beta(S)^{2}-T \beta(T)^{2}\right\|_{2} \leq\|S-T\|_{2} .
$$

However, for the usual operator norm we have

$$
\left\|S \beta(S)^{2}-T \beta(T)^{2}\right\| \leq \frac{5}{4}\|S-T\| .
$$

To prove this inequality it is sufficient to assume that $S$ and $T$ are selfadjoint. In this case we have

$$
\begin{aligned}
S \beta(S)^{2}-T \beta(T)^{2}= & S\left(1+S^{2}\right)^{-1}-T\left(1+T^{2}\right)^{-1} \\
= & \left(1+S^{2}\right)^{-1}\left(S\left(1+T^{2}\right)-\left(1+S^{2}\right) T\right)\left(1+T^{2}\right)^{-1} \\
= & \left(1+S^{2}\right)^{-1}(S-T)\left(1+T^{2}\right)^{-1} \\
& +\left(1+S^{2}\right)^{-1} S(T-S) T\left(1+T^{2}\right)^{-1}
\end{aligned}
$$

Since $\left\|\left(1+T^{2}\right)^{-1}\right\| \leq 1$ and $\left\|T\left(1+T^{2}\right)^{-1}\right\| \leq \frac{1}{2}$, it follows that $\| S \beta(S)^{2}-$ $T \beta(T)^{2}\left\|\leq \frac{5}{4}\right\| S-T \|$.

Finally, we remark that this inequality is also true for every unitarily invariant norm.

\section{REFERENCES}

1. T. Ando, Topics on operator inequalities, preprint, Research Institute of Applied Electricity, Hokkaido, Sapporo, Japan.

2. Comparison of norms ||$|f(a)-f(b)|||$ and ||$|f(|A-B|)|||$, Math. Z. 197 (1988), 403-409.

3. J. Arazy, More on convergence in unitary matrix spaces, Proc. Amer. Math. Soc. 83 (1981), 44-48.

4. R. Bhatia, Perturbation inequalities for the absolute value map in norm ideals of operators, J. Operator Theory 19 (1988), 129-136.

5. R. Bhatia and F. Kittaneh, On some perturbation inequalities for operators, Linear Algebra Appl. 106 (1988), 271-279.

6. __ Norm inequalities for partitioned operators and an application, preprint.

7. E. B. Davies, Lipschitz continuity of functions of operators in the Schatten classes, J. London Math. Scc. 37 (1988), 148-157.

8. T. Kato, Perturbation theory for linear operators, 2nd ed., Springer-Verlag, New York, 1976.

9. W. E. Kaufman, Representing a closed operator as a quotient of continuous operators, Proc. Amer. Math. Soc. 72 (1978), 531-534.

10. __ Closed operator and pure contractions in Hilbert space, Proc. Amer. Math. Soc. 87 (1983), 83-87. 
11. _ A stronger metric for closed operators in Hilbert space, Proc. Amer. Math. Soc. 90 (1984), 83-87.

12. F. Kittaneh, On Lipschitz functions of normal operators, Proc. Amer. Math. Soc. 94 (1985), 416-418.

13. _ Inequalities for the Schatten p-norm. II, Glasgow Math. J. 29 (1987), 99-104.

14. __ Inequalities for the Schatten p-norm. IV, Commun. Math. Phys. 106 (1986), 581-585.

15. _ On the continuity of the absolute value map in the Schatten classes, Linear Algebra Appl. 118 (1989), 61-68.

16. F. Kittaneh and H. Kosaki, Inequalities for the Schatten p-norm. V, Publ. Res. Inst. Math. Sci. Kyoto Univ. 23 (1987), 433-443.

17. R. Schatten, Norm ideals of completely continuous operators, Springer-Verlag, Berlin, 1960.

Department of Mathematics, Kuwait University, Kuwait 